\title{
Erratum to: Structuprint: a scalable and extensible tool for two-dimensional representation of protein surfaces
}

Dimitrios Georgios Kontopoulos ${ }^{1 *}$, Dimitrios Vlachakis ${ }^{2^{*}}$, Georgia Tsiliki $^{3}$ and Sofia Kossida ${ }^{4}$

Due to a formatting error during the production process of this article [1], equations 4 and 5 were published incorrectly in the PDF of the article [1]. The equations were, however, correctly presented in the HTML version of the article. Equations 4 and 5 are correctly included in this erratum.

The publisher takes full responsibility for the errors which occurred and sincerely apologises for the inconvenience caused.

$$
\begin{gathered}
\mathbf{w}_{\boldsymbol{i}}=\left(x_{i}^{\prime \prime}, y_{i}^{\prime \prime}, z_{i}^{\prime \prime}\right)=\frac{\text { radius }}{\sqrt{x_{i}^{\prime 2}+y_{i}^{\prime 2}+z_{i}^{\prime 2}}} \cdot \mathbf{v}_{i}^{\prime} \\
\text { latitude }_{i}=\tan ^{-1} \frac{z_{i}^{\prime \prime}}{\sqrt{x_{i}^{\prime \prime}+y_{i}^{\prime 2}}} \\
\text { longitude }_{i}=\tan ^{-1} \frac{y_{i}^{\prime \prime}}{x_{i}^{\prime \prime}}
\end{gathered}
$$

\footnotetext{
Author details

${ }^{1}$ Department of Life Sciences, Imperial College London, Silwood Park Campus, Ascot, UK. ${ }^{2}$ Bioinformatics \& Medical Informatics Team, Biomedical Research Foundation, Academy of Athens, Athens, Greece. ${ }^{3}$ School of Chemical Engineering, National Technical University of Athens, Athens, Greece. ${ }^{4} \mathrm{IMGT}{ }^{\oplus}$, The International ImMunoGeneTics Information System ${ }^{\oplus}$, Université de Montpellier, Laboratoire d'ImmunoGénétique Moléculaire LIGM, UPR CNRS 1142, Institut de Génétique Humaine, Montpellier, France.

Received: 4 March 2016 Accepted: 4 March 2016

Published online: 11 March 2016

\section{Reference}

1. Kontopoulos et al. Structuprint: a scalable and extensible tool for twodimensional representation of protein surfaces. BMC Structural Biology. 2016 Feb 24; 16:4. doi: 10.1186/s12900-016-0055-7.

* Correspondence: d.kontopoulos13@imperial.ac.uk; dvlachakis@bioacademy.gr The online version of the original article can be found under doi:10.1186/ s12900-016-0055-7.

'Department of Life Sciences, Imperial College London, Silwood Park Campus, Ascot, UK

${ }^{2}$ Bioinformatics \& Medical Informatics Team, Biomedical Research

Foundation, Academy of Athens, Athens, Greece
}

Submit your next manuscript to BioMed Central and we will help you at every step:

- We accept pre-submission inquiries

- Our selector tool helps you to find the most relevant journal

- We provide round the clock customer support

- Convenient online submission

- Thorough peer review

- Inclusion in PubMed and all major indexing services

- Maximum visibility for your research

Submit your manuscript at

www.biomedcentral.com/submit \section{西}

